\title{
In vivo efficacy of artemether-lumefantrine against uncomplicated Plasmodium falciparum malaria in Dembia District, northwest Ethiopia
}

This article was published in the following Dove Press journal:

Therapeutics and Clinical Risk Management

16 February 2017

Number of times this article has been viewed

\author{
Tekalign Deressa' \\ Mengistu Endris Seid ${ }^{1,2}$ \\ Wubet Birhan' \\ Yetemwork Aleka' \\ Biniam Mathewos Tebeje ${ }^{1,3}$ \\ 'School of Biomedical and Laboratory \\ Sciences, College of Medicine and \\ Health Sciences, University of Gondar, \\ Gondar, Ethiopia; ${ }^{2}$ Australian Institute \\ Tropical Health and Medicine, James \\ Cook University, Cairns, Australia; \\ 3Molecular Parasitology Laboratory, \\ Queensland Institute of Medical \\ Research, Brisbane, Australia
}

Background: Artemether-lumefantrine (AL) has been used as a first-line treatment for uncomplicated Plasmodium falciparum malaria in Ethiopia since 2004. Antimalarial drug resistance is one of the major obstacles for malaria control and curtails the lifespan of several drugs. Thus, continued monitoring of the efficacy of $\mathrm{AL}$ is of great public health importance in malaria endemic areas.

Objective: This study aimed to investigate the therapeutic efficacy and safety of AL for the treatment of uncomplicated P. falciparum malaria in the Dembia district, northwest Ethiopia. Methods: A prospective study was conducted from April 2015 to February 2016 at Kola Diba Health Center (KHC) in the Dembia district to determine the therapeutic efficacy and safety of AL for the treatment of uncomplicated $P$. falciparum monoinfection. Patients were treated with the six-dose regimen of AL over 3 days and followed up for 28 days as per the World Health Organization protocol.

Results: Of the total 80 patients enrolled in the AL efficacy study, 75 patients completed the 28 days follow-up. None of the participants reported major adverse events. No early treatment failure or late clinical failure were observed during the study, but there were $6(8.0 \%)$ late parasitological failures. The uncorrected per protocol cure rate of AL was 92.0 (95\% CI: 85.7-98.3). Treatment with AL cleared parasitemia and fever in $>95 \%$ of the patients by day 3 .

Conclusion: This study showed that AL is well tolerated and remains efficacious for treatment of uncomplicated P. falciparum malaria in northwest Ethiopia. However, the observed late parasitological failures in this study are of a concern and warrant continued monitoring of drug efficacy as per the World Health Organization recommendations.

Keywords: artemether-lumefantrine, uncomplicated Plasmodium falciparum, Ethiopia, malaria, antimalarial efficacy

\section{Background}

Malaria caused by five species of Plasmodium parasites remains a substantial health burden globally. The World Health Organization estimates $\sim 214$ million malaria cases and 438,000 deaths from this disease in the year 2015. More than $88 \%$ of the world malaria cases and $90 \%$ of malaria-related deaths occur in sub-Saharan African countries. ${ }^{1,2}$

In Ethiopia, malaria is the leading cause of morbidity and mortality, with $>5$ million cases and 70,000 deaths each year. About 75\% of the country is malarious, and $60 \%$ of its population is at risk of this infection. ${ }^{3,4}$ Plasmodium falciparum and Plasmodium vivax are the predominant malaria parasites in Ethiopia, with the former species being accountable for $\sim 65 \%-75 \%$ of reported malaria cases and associated with complicated clinical manifestations.,
Correspondence: Tekalign Deressa School of Biomedical and Laboratory Sciences, College of Medicine and Health Sciences, University of Gondar, PO Box 196, Gondar, Ethiopia Email tekalign09@gmail.com 
Antimalarial drug resistance is one of the major obstacles for malaria control and curtails the lifespan of several drugs. Following a widespread failure of chloroquine (CQ) and sulfadoxine-pyrimethamine (SP), most malaria endemic countries had shifted their malaria treatment policy to artemisinin-based combination therapy (ACT). Ethiopia has adopted artemether-lumefantrine (AL), an ACT antimalarial, as the first-line drug for the treatment of uncomplicated $P$. falciparum malaria in July 2004..$^{5}$ A baseline study conducted in 2004 to assess the efficacy of AL against P. falciparum malaria has shown $\sim 100 \%$ cure rate. ${ }^{6}$ Subsequent studies from different parts of the country also reported the high efficacy of AL with a treatment failure rate not $>5 \%$, following the 28-day in vivo test protocol..$^{7-11}$

However, increased tolerance of the parasites to artemisinin-derived antimalarial drugs has already been reported from southeast Asian, the historical epicenter for the start of antimalarial drug resistance. ${ }^{12-14}$ Some studies also reported the presence of confirmed resistance in this area. ${ }^{14,15}$ However, there is no such report from sub-Saharan African countries. ${ }^{12}$ Nevertheless, the ACT antimalarials such as AL, amodiaquine-artesunate, and dihydroartemisinin-piperaquine are now being deployed intensively in African countries. ${ }^{16-19}$ Given drug pressure is one of the important drivers for selection of drug-resistant Plasmodium parasites, continuous monitoring of usage and efficacy of these antimalarials has a vital significance for malaria control programs. With this regard, the WHO recommends strict monitoring of efficacies of antimalarials every 2 years for the early detection of resistance development and rapid identification of its new foci. Such data are desirable both for immediate containment and prevention of antimalarial resistance and for timely updates of treatment policy. To this end, our study aims to investigate the therapeutic efficacy and safety of AL against uncomplicated $P$. falciparum malaria in Dembia district of North Gondar Zone, northwest Ethiopia.

\section{Methods}

\section{Study design, period, and site}

A prospective study was conducted from April 2015 to February 2016 at Kola Diba Health Center (KHC) of the Dembia district of the North Gondar Zone, northwest Ethiopia. Dembia is located at $729 \mathrm{~km}$ north of the country's capital Addis Ababa, close to Lake Tana (the largest lake in Ethiopia). The district covers an area of 1,270 km with the altitude ranging between 1,750 and 2,100 $\mathrm{m}$ above sea level. It has an estimated population of $\sim 271,053 .{ }^{19}$ The district is characterized by high malaria transmission rate (peak from October to December) and extensive deployment of antimalarials, which might select for drug-resistant parasite species. $P$. falciparum and $P$. vivax were accountable for $75 \%$ and $25 \%$ of malaria cases in the area, respectively. ${ }^{20}$

\section{Sample size determination}

The sample size was determined based on the WHO standard protocol for evaluating antimalarial therapeutic efficacy. ${ }^{21,22}$ Considering the anticipated population proportion of clinical failure of 5\%, with $95 \%$ confidence interval (CI), $5 \%$ precision, and $10 \%$ loss to follow-up, 80 patients were enrolled.

\section{Patients, treatment, and follow-up}

The study subjects were selected based on the following inclusion criteria: age $>6$ months, confirmed infection with P. falciparum, initial density of 1,000-100,000 asexual parasite $/ \mu \mathrm{L}$, and axillary temperature $\geq 37.5^{\circ} \mathrm{C} /$ history of fever. Exclusion criteria were pregnancy or breastfeeding, severe co-morbidity, co-infections of $P$. falciparum with other Plasmodium species, severe malnutrition, and danger signs such as prostration, coma, multiple convulsions, respiratory distress, pulmonary edema, jaundice, hyperparasitemia, and severe anemia. $P$. falciparum-positive cases were treated with six oral doses of AL (Novartis, Geneva, Switzerland) scaled by age/weight according to the national policy. To facilitate the follow-up, patients within $20 \mathrm{~km}$ distance from $\mathrm{KHC}$ were enrolled into the study. All study participants were followed on days $1,2,3,7,14,21$, and 28 , and on any other day if they felt ill. ${ }^{21,22}$

\section{Clinical and laboratory procedures}

The screening was done by clinicians to identify patients meeting the inclusion criteria. Data including age, sex, and complete medical history, including prior and concomitant medications, were recorded at baseline by health personnel in an outpatient setting. Similarly, axillary temperature was measured at baseline and on the subsequent visits. Fingerprick blood sample was collected from each patient. Thick and thin blood smears were prepared as outlined in malaria microscopy using $10 \%$ Giemsa stain ( $\mathrm{pH} 7.2$ ) for $10 \mathrm{~min}^{23}$ The blood films were prepared for microscopy before treatment on day 0 and on days $1,2,3,7,14,21$, and 28 .

Parasitemia was estimated by counting the number of asexual parasites against a number of leukocytes (200+) in the thick blood film, based on a putative mean count of 8,000 leukocytes per $\mu \mathrm{L}$. The following formula was used to determine parasites, density ${ }^{22}$ :

Parasitemia/ $\mu \mathrm{L}$ $=$ Number of parasites $\times 8000 /$ Number of leukocytes 
A blood slide was declared negative when the examination of 100 fields did not show the presence of asexual forms of $P$. falciparum.

\section{Outcomes}

The WHO protocol was used to assess the therapeutic efficacy of AL against uncomplicated $P$. falciparum malaria. ${ }^{22}$ According to the definition of WHO, early treatment failure (ETF) is the development of danger signs on days 1, 2, or 3 with insufficient parasitemia clearance on day 3 ( $\geq 25 \%$ of count on day 0 ). Patients with danger signs after day 3 or history of fever on any day from day 4 to 28 were grouped as late clinical failure (LCF), whereas those with parasitemia on any day from day 7 to 28 were classified as late parasitological failure (LPF). If parasitemia was absent on day 28 , it was considered as adequate clinical and parasitological response (ACPR).

The secondary outcomes were fever clearance rate: proportion of patients who have fever cleared at day 1, 2, and 3 . Parasite clearance rate: proportion of patients with negative thick blood film smears on days 1, 2, and 3. Gametocyte carriage: proportion of patients with gametocytes during the course of the study.

\section{Statistical analysis}

Data were entered, cleaned, and analyzed using SPSS version 20 statistical package software (SPSS, Inc., Chicago, IL, USA). Descriptive statistics such as percentages, mean, median, standard deviation, and range was applied as appropriate. The efficacy assessment was performed by modified intention-to-treat (ITT) and per protocol (PP) analysis. The ITT population was used for analysis of variables, primary and secondary efficacy endpoints. The PP employed Kaplan-Meier used for analysis of primary outcomes (ETF, LCF, LPF, and ACPR). $P$-values $<0.05$ were considered statistically significant.

\section{Ethical clearance}

Ethical clearance was obtained from the institutional ethical review board of the University of Gondar. A written informed consent was obtained from all study participants. In the case of children under the age of 18 years, consent was obtained from a parent or legal guardian.

\section{Results}

\section{Characteristics of the study participants}

A total of 80 consented patients who fulfilled the inclusion criteria were enrolled in the study. Of these, 57 of $80(71.2 \%)$ were male and 23 of $80(28.8 \%)$ were female. The median
Table I Baseline characteristics of study subjects at Kola Diba Health Center, northwest Ethiopia, April 2015-February 2016

\begin{tabular}{|c|c|}
\hline Characteristics & Frequency (\%) \\
\hline \multicolumn{2}{|l|}{ Gender } \\
\hline Male & 57 (7I.2) \\
\hline Female & $23(28.8)$ \\
\hline \multicolumn{2}{|l|}{ Age group (years) } \\
\hline $0-15$ & $25(3 \mid .2)$ \\
\hline$>15$ & $55(68.8)$ \\
\hline \multicolumn{2}{|l|}{ Age (years) } \\
\hline Mean (SD) & $22.36(11.3)$ \\
\hline Median (IQR) & $20.0( \pm 13.0)$ \\
\hline Range (min-max) & $48(2-50)$ \\
\hline \multicolumn{2}{|l|}{ Temperature $\left({ }^{\circ} \mathrm{C}\right)$, day 0} \\
\hline Mean temperature $(\mathrm{SD})$ & $37.86(0.38)$ \\
\hline Range (min-max) & $37.0-38.6$ \\
\hline \multicolumn{2}{|l|}{ Parasitemia $(\mu \mathrm{L})$, day 0} \\
\hline Mean (geometric) parasitemia & 8377.78 \\
\hline \multicolumn{2}{|l|}{ Parasitemia grade, day 0} \\
\hline Moderate $(<10,000 / \mu \mathrm{L})$ & $48(60.0)$ \\
\hline Severe $(\geq 10,000 / \mu \mathrm{L})$ & $32(40.0)$ \\
\hline
\end{tabular}

Abbreviations: IQR, interquartile range; SD, standard deviation.

age (interquartile range [IQR]) of the patients was 20.0 (13.0). A little over two-thirds (68.8\%) of the study participants were above the age of 15 years. The mean temperature (SD) and parasitemia (geometric mean) of the patients were $37.86(0.38)$ and 8377.8 , respectively (Table 1$)$. The mean parasite density was higher among the participants below the age of 5 years $(41.05$ parasites $/ \mu \mathrm{L})$ than the remaining groups ( $P=0.03$ ) (data not shown). However, there were no significant differences in the baseline mean temperature of all age groups (Table 2).

\section{Treatment outcomes}

Of the total 80 patients enrolled in the AL efficacy study, 75 patients completed the 28 days follow-up (Figure 1). Five patients (6.3\%) were censored, three lost to follow-up, one missed the evening dose, and one was infected with $P$. vivax on day 21 . None of the study participants reported severe adverse effects during the follow-up except that 2 of $80(2.5 \%)$ participants reported common complaints like nausea and coughing. Furthermore, there were no ETFs and LCFs, but there were six (8.0\%) LPFs.

Table 2 Baseline characteristics of the study participants at Kola Diba Health Center, stratified by age group

\begin{tabular}{|c|c|c|}
\hline \multirow[t]{2}{*}{ Characteristics } & \multicolumn{2}{|c|}{ Age groups (years) } \\
\hline & $0-15(95 \% \mathrm{Cl})$ & $>I 5(95 \% \mathrm{Cl})$ \\
\hline Mean $\mathrm{T}\left({ }^{\circ} \mathrm{C}\right)$ & $37.94(37.8-38.1)$ & $37.83(37.7-37.9)$ \\
\hline MPD $(\times 1,000)$ & $20.43(10.8-30.1)$ & $12.28(7.80-16.76)$ \\
\hline
\end{tabular}

Abbreviations: $\mathrm{Cl}$, confidence interval; MPD, mean parasite density; $\mathrm{T}$, temperature. 


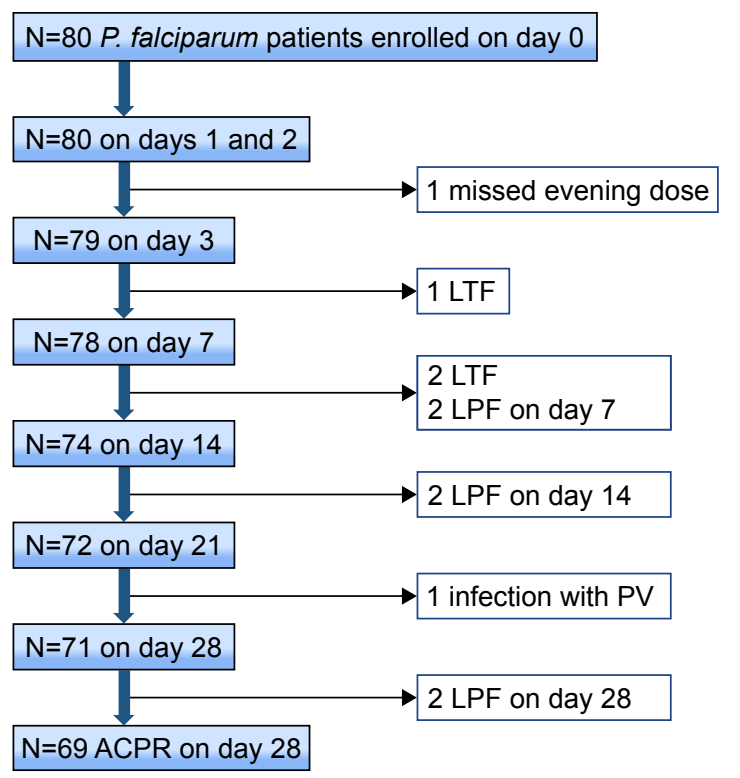

Figure I Flowchart depicting follow-up and outcomes of the study participants. Abbreviations: ACPR, adequate clinical and parasitological response; LPF, late parasitological failure; LTF, lost to follow up; PV, Plasmodium vivax.

Of these six patients, $83.3 \%$ (5/6) were male, and $16.7 \%$ (1/6) was under 5 years old. The median age of these patients was 15 years (IQR \pm 10.5$)$, and the range was $2-20$. In terms of parasitemia grade, $66.7 \%(4 / 6)$ had severe parasitemia at the baseline, and the mean (SD) parasitemia was 33,900 $(15,863)$. Three of the four patients who showed LPF between days 7 and 14 had severe parasitemia grade at the baseline. But, all of these patients were asymptomatic. The uncorrected cure rate for the PP and ITT analyses was 92.0 (95\% CI: 85.7-98.3) and 86.3 (95\% CI: 78.5-93.9), respectively (Table 3).

\section{Secondary treatment outcomes}

Treatment with AL displayed a rapid parasite clearance and fever resolution rates (Table 4). Of all the study participants,

Table 3 Treatment outcome of the study subjects after followup for 28 days, Kola Diba Health Center, northwest Ethiopia

\begin{tabular}{ll}
\hline Outcomes & Value (\%) \\
\hline Censored & $5(6.3)$ \\
Lost to follow-up & $3(3.8)$ \\
Missed evening dose & $\mathrm{I}(1.3)$ \\
$\quad$ Infection with different species & $\mathrm{I}(\mathrm{I} .3)$ \\
Failure & \\
$\quad$ Early treatment failure & $0(0.0)$ \\
Late clinical failure & $0(0.0)$ \\
Late parasitological failure & $6(8.0)$ \\
ACPR-uncorrected & $69(86.3)$ \\
Cure rate - per protocol $(95 \% \mathrm{Cl})$ & $92.0(85.7-98.3)$ \\
Cure rate - intention to treat $(95 \% \mathrm{Cl})$ & $86.3(78.5-93.9)$ \\
\hline
\end{tabular}

Abbreviations: ACPR, adequate clinical and parasitological response; $\mathrm{Cl}$, confidence interval.
Table 4 Secondary outcomes of the study participants stratified by age

\begin{tabular}{llll}
\hline Outcomes & \multicolumn{2}{l}{ Age groups (years) } & \multirow{2}{*}{ Total } \\
\cline { 2 - 3 } & $\mathbf{0 - 1 5}$ & $>$ I5 & \\
\hline Fever, n/N (\%) & & & \\
Day I & $10 / 25(40)$ & $20 / 55(36.4)$ & $30 / 80(37.5)$ \\
Day 2 & $1 / 25(4.0)$ & $4 / 55(7.3)$ & $5 / 80(6.3)$ \\
Day 3 & $0 / 25(0.0)$ & $2 / 55(3.6)$ & $2 / 80(2.5)$ \\
Parasitemia, n/N (\%) & & \\
Day I & $9 / 25(36.0)$ & $17 / 55(30.9)$ & $26 / 80(32.5)$ \\
Day 2 & $3 / 25(12.0)$ & $9 / 55(16.4)$ & $12 / 80(15.0)$ \\
Day 3 & $1 / 25(4.0)$ & $3 / 55(5.5)$ & $4 / 80(5.0)$ \\
Gametocytes, n/N (\%) & & \\
Day 0 & $0 / 25(0.0)$ & $2 / 55(3.6)$ & $2 / 80(2.5)$ \\
Day I & $0 / 25(0.0)$ & $3 / 55(5.5)$ & $3 / 80(3.8)$ \\
Day 2 & $0 / 25(0.0)$ & $2 / 55(3.6)$ & $2 / 80(2.5)$ \\
Day 3 & $0 / 25(0.0)$ & $1 / 55(1.8)$ & $1 / 80(1.3)$ \\
Day 7 & $0 / 22(0.0)$ & $0 / 55(0.0)$ & $0 / 80(0.0)$ \\
\hline
\end{tabular}

$67.5 \%, 85 \%$, and $95 \%$ cleared parasitemia on days 1,2 , and 3 , respectively. Fever was noted only in $6.3 \%$ and $2.5 \%$ on days 2 and 3, respectively. All of the patients with fever on the above-mentioned days were with high parasitemia grades on the day of enrollment. Gametocytes were observed in 2 of the $80(2.5 \%)$ of the study participants on the day of enrollment and in 1 of $80(1.3 \%)$ patients on day 1 . Patients with gametocytes on day 0 cleared the gametocytes on days 2 and 3. No gametocytes were observed after day 3 . In terms of the age group, all gametocyte cases were noted among patients over the age of 16 years.

\section{Discussion}

In Ethiopia, AL (a.k.a. Coartem ${ }^{\circledR}$ ) has been used as a first-line drug for treatment of uncomplicated $P$. falciparum malaria and mixed infection since July 2004. However, data on AL efficacy from Ethiopia especially from the northwest part are scarce. ${ }^{7,8}$ The emergence of $P$. falciparum resistance to ACT drugs in southeast Asia threatens the current firstline treatment for severe malaria. ${ }^{14,15}$ Development of drug resistance is a consequence of intensive use/misuse of antimalarials, including poor treatment practices, inadequate patient adherence, use of artemisinin-based monotherapies or substandard forms of antimalarial drugs, and various host-parasite interactions..$^{13,24,25}$ Thus, it is critical to put a stringent antimalarial efficacy monitoring system in place to ensure early detection of changes in parasite resistance and to inform malaria control policymakers.

Our study showed that AL remains efficacious for the treatment of malaria by P. falciparum. The PP cure rate of AL $(92.0 \%)$ observed in this study was in line with $92.5 \%$ 
in northwest Ethiopia, ${ }^{7} 93 \%$ in Togo, ${ }^{18}$ and $92.5 \%$ in Bastar, India. ${ }^{26}$ This finding was also within a range with $96.3 \%$ cure rate in Kersa, ${ }^{9} 97.6 \%$ in Metehara ${ }^{10}$ and $94.4 \%$ in southwest Ethiopia. ${ }^{11}$ The high AL efficacy observed in this study could be in part ascribed to the fact that distribution of this antimalarial is restricted to the government sectors to minimize circulations of substandard counterfeit drugs. ${ }^{27}$ Further, continuous follow-up of AL usage by health extension workers might have also improved patients adherence to the treatment regimen.

Delays in parasite clearance time have been used as an indicator for suspected artemisinin resistance in P. falciparum. ${ }^{14,15}$ In this study, treatment with AL displayed rapid parasite and fever clearance rates. All of the patients but two were cleared of the fever on day 3 . Similarly, $\sim 95 \%$ and $98 \%$ of the patients had cleared parasitemia and gametocytes on day 3 of treatment, respectively, indicating that AL was effective in reduction of the parasite biomass. This finding was consistent with therapeutic efficacy values reported by a number of previous studies. , $^{7,-30}$

In terms of tolerability, AL was well tolerated by all study participants including the children who were treated by this drug. This was consistent with the previous reports. ${ }^{7,17}$ No adverse events were noted in children as well as adolescents who took part in this study, but two adults reported the well-characterized AL side effects such as nausea and coughing.

Given that most of the patients with a delayed parasites clearance can still be cured with $\mathrm{AL}$, the treatment failure observed in this study might not necessarily mean an emergence of AL-resistant strains. This is because a number of factors like host immunity, re-infection, low drug level in blood, and recrudescence cases could influence the outcomes of a therapeutic efficacy test. ${ }^{31-33}$ Thus, we recommend further investigations with definitive confirmatory tests for AL resistance such as in vitro drug susceptibility tests, molecular markers for drug resistance or to rule out recrudescent/ reinfection cases, and pharmacokinetics.

Nevertheless, the re-emergence of parasitemia as early as day 7 after the start of treatment and a relatively high proportion of patients $(5 \%)$ with parasitemia on day 3 observed in this study might have potential clinical implications.

\section{Conclusion}

The results of this study demonstrate that AL is well tolerated and remains efficacious for treatments of uncomplicated $P$. falciparum malaria in northwest Ethiopia. However, the delayed parasitological failures observed in this study suggest the need for continued monitoring of drug efficacy as per the WHO recommendations.

\section{Acknowledgments}

We are very thankful to the nurses and diagnostic laboratory staffs of Kola Diba Health Center for their unreserved support during patients' selection and follow-up. We also extend our gratitude to all our study participants for being volunteers to participate in this study.

\section{Author contributions}

TD designed the experiment and performed data collection and analysis; MES, WB, and BMT participated in designing the study and performing the data collection and statistical analysis; YA performed microscopy and helped in drafting the paper; and TD wrote the manuscript. All authors contributed toward data analysis, drafting and revising the paper and agree to be accountable for all aspects of the work.

\section{Disclosure}

The authors report no conflicts of interest in this work.

\section{References}

1. World Health Organization 2015. World malaria report 2015. Available from: http://www.who.int/malaria/publications/world-malaria-report2015/en/. Accessed October 25, 2016.

2. World Health Organization. Guidelines for the treatment of malaria. Geneva, Switzerland: World Health Organization; 2011.

3. WHO. World Health Organization regional office for Africa. Malaria in Ethiopia. Available from: http://www.afro.who.int/en/ethiopia/ country-programmes/topics/4580-ethiopia-malaria.html. Accessed December 29, 2016.

4. Federal Democratic Republic of Ethiopia MoH. Malaria epidemiological profile. Available from: http://www.moh.gov.et/malaria. Accessed December 29, 2016.

5. Federal Democratic Republic of Ethiopia MoH, Guideline for Malaria Epidemic Prevention and Control in Ethiopia. Ethiopia: Addis Ababa; 2004.

6. Jima D, Tesfaye G, Medhin A, Kebede A, Argaw D, Babaniyi O. Safety and efficacy of artemether-lumefantrine in the treatment of uncomplicated falciparum malaria in Ethiopia. East Afr Med J. 2005; 82(8):387-390.

7. Getnet G, Fola AA, Alemu A, Getie S, Fuehrer HP, Noedl H. Therapeutic efficacy of artemether-lumefantrine for the treatment of uncomplicated Plasmodium falciparum malaria in Enfranze, north-west Ethiopia. Malar J. 2015;14:258.

8. Wudneh F, Assefa A, Nega D, et al. Open-label trial on efficacy of artemether/lumefantrine against the uncomplicated Plasmodium falciparum malaria in Metema. Ther Clin Risk Manag. 2016;12:1293-1300.

9. Assefa A, Kassa M, Tadese G, Mohamed H, Animut A, Mengesha T. Therapeutic efficacy of artemether/lumefantrine $\left(\right.$ Coartem $\left.^{\circledR}\right)$ against Plasmodium falciparum in Kersa, South West Ethiopia. Parasit Vectors. 2010;3(1):1.

10. Nega D, Assefa A, Mohamed H, et al. Therapeutic efficacy of artemether-lumefantrine (Coartem) in treating uncomplicated P. falciparum malaria in Metehara, Eastern Ethiopia: regulatory clinical study. PLoS One. 2016;11(4):e0154618. 
11. Mekonnen SK, Medhin G, Berhe N, Clouse RM, Aseffa A. Efficacy of artemether-lumefantrine therapy for the treatment of uncomplicated Plasmodium falciparum malaria in Southwestern Ethiopia. Malar J. 2015;14:317.

12. Ashley EA, Dhorda M, Fairhurst RM, et al. Spread of artemisinin resistance in Plasmodium falciparum malaria. N Engl J Med. 2014; 371(5):411-423.

13. WHO 2013. Emergency response to artemisinin resistance in the Greater Mekong Sub-region. Available from: http://www.who.int/malaria/ publications/atoz/9789241505321/en/. Accessed October 18, 2015.

14. Dondorp AM, Nosten F, Yi P, Das D, et al. Artemisinin resistance in Plasmodium falciparum malaria. N Engl J Med. 2009;361:455-467.

15. Noedl H, Se Y, Schaecher K, Smith BL, Socheat D, Fukuda MM; Artemisinin Resistance in Cambodia 1 (ARC1) Study Consortium. Evidence of artemisinin-resistant malaria in western Cambodia. $N$ Engl J Med. 2008;359(24):2619-2620.

16. Yeka A, Lameyre V, Afizi K, et al. Efficacy and safety of fixed-dose artesunate-amodiaquine vs artemether-lumefantrine for repeated treatment of uncomplicated malaria in Ugandan children. PLoS One. 2014; 9(12):e113311.

17. de Wit M, Funk AL, Moussally K, et al. In vivo efficacy of artesunateamodiaquine and artemether-lumefantrine for the treatment of uncomplicated falciparum malaria: an open-randomized, non-inferiority clinical trial in South Kivu, Democratic Republic of Congo. Malar J. 2016;15:455.

18. Dorkenoo MA, Barrette A, Agbo YM, et al. Surveillance of the efficacy of artemether-lumefantrine and artesunate-amodiaquine for the treatment of uncomplicated Plasmodium falciparum among children under five in Togo, 2005-2009. Malar J. 2012;11:338.

19. Central Statistical Agency, 2007. Summary and Statistical Report of the 2007 Population and Housing Census Results. December; 2008. Ethiopia: Addis Ababa.

20. Alemu A, Muluye D, Mihret M, Adugna M, Gebeyaw M. Ten year trend analysis of malaria prevalence in Kola Diba, North Gondar, Northwest Ethiopia. Parasit Vectors. 2012;5:173.

21. World Health Organization. "Susceptibility of Plasmodium Falciparum to Antimalarial drugs." Report on global monitoring 1996-2004 WHO/HTM/mal/2005.11.3., Geneva, Switzerland: World Health Organization; 2005.

22. WHO. Method for Surveillance of Antimalarial Drug Efficacy. Geneva, Switzerland: World Health Organization; 2009 Available from: http:// www.who.int/malaria/resistance. Accessed on October 18, 2015.
23. WHO. Basic Malaria Microscopy-second edition: Part I Learner's guide; Part II Tutor's guide. Geneva, Switzerland: World Health Organization; 2010.

24. Payne D. Spread of chloroquine resistance in Plasmodium falciparum. Parasitol Today. 1987;3(8):241-246.

25. World Health Organization. Global Malaria Control and Elimination: Report of a Meeting on Containment of Artemisinin Tolerance. WHO Report. Geneva, Switzerland: WHO; 2008. Available from: whqlibdoc. who.int/publications/2008/9789241596817_eng.pdf. Accessed October 18, 2015.

26. Bharti PK, Shukla MM, Ringwald P, et al. Therapeutic efficacy of artemether-lumefantrine for the treatment of uncomplicated Plasmodium falciparum malaria from three highly malarious states in India. Malar J. 2016;15(1):498.

27. Federal Democratic Republic of Ethiopia, Ministry of Health. Malaria Diagnosis and Treatment Guidelines for Health Workers in Ethiopia. 2nd ed. Ethiopia: Addis Ababa Minist Health; 2004.

28. Hwang J, Alemayehu BH, Hoos D, et al. In vivo efficacy of artemetherlumefantrine against uncomplicated Plasmodium falciparum malaria in Central Ethiopia. Malar J. 2011;10:209.

29. Dorsey G, Staedke S, Clark TD, Njama-Meya D, et al. Combination therapy for uncomplicated falciparum malaria in Ugandan children: a randomized trial. JAMA. 2007;297(20):2210-2219.

30. Arinaitwe E, Sandison TG, Wanzira H, et al. Artemether-lumefantrine versus dihydroartemisinin-piperaquine for falciparum malaria: a longitudinal, randomized trial in young Ugandan children. Clin Infect Dis. 2009;49(11):1629-1637.

31. Ezzet F, van Vugt M, Nosten F, Looareesuwan S, White NJ. Pharmacokinetics and pharmacodynamics of lumefantrine (benflumetol) in acute falciparum malaria. Antimicrob Agents Chemother. 2000;44(3): 697-704.

32. Djimde AA, Doumbo OK, Traore O, et al. Clearance of drug-resistant parasites as a model for protective immunity in $P$. falciparum malaria. Am J Trop Med Hyg. 2003;69(5):558-563.

33. White NJ. Why is it that antimalarial drug treatments do not always work? Ann Trop Med Parasitol. 1998;92(4):449-458.
Therapeutics and Clinical Risk Management

\section{Publish your work in this journal}

Therapeutics and Clinical Risk Management is an international, peerreviewed journal of clinical therapeutics and risk management, focusing on concise rapid reporting of clinical studies in all therapeutic areas, outcomes, safety, and programs for the effective, safe, and sustained use of medicines. This journal is indexed on PubMed Central, CAS,

\section{Dovepress}

EMBase, Scopus and the Elsevier Bibliographic databases. The manuscript management system is completely online and includes a very quick and fair peer-review system, which is all easy to use. Visit http://www.dovepress.com/testimonials.php to read real quotes from published authors. 